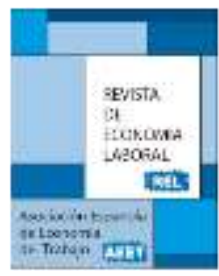

\title{
MERCADOS INTERNOS DE TRABAJO EN LOS GRUPOS ECONÓMICOS DE MÉXICO. UN ESTUDIO DE CASO
}

\author{
Rubén Chavarín ${ }^{1}$ y Gerardo Ríos-Almodóvar \\ Universidad de Guadalajara
}

Recibido Abril 2018; Aceptado Junio 2018

\section{Resumen}

El presente estudio documenta el uso de un mercadointerno de trabajo en la cadena de tiendas de conveniencia Oxxo, dentro del grupo económico FEMSA, uno de los más grandes y antiguos de México, y con operaciones en otros diez países de América Latina. Encontramos que el mercadointerno de Oxxo-FEMSA cumplecon las siguientescondiciones: a) promueve el ascenso y movilidad de sus propios empleados para cubrir las vacantes que surgen en los escalafones superiores de su estructura jerárquica, incluyendo transferencias a otros negocios del grupo, convirtiéndolo en un mercado cuasi-interno de trabajo; $b$ ) incluye organizaciones establecidas por el propio grupo económico para la formación del capital humano, con antecedentes significativos desde hace décadas; y c) presenta cierta evidencia de diferencias en los salarios que perciben los trabajadores, comparados con aquellos de otras opciones laborales en el mismo mercado. El estudio de caso también aporta evidencia de la articulación de distintas estructuras del mercado de trabajo dentro del mismo grupo económico, ya que el uso del mercado cuasi-interno de trabajo se combina con subcontratación de personal y participación de trabajadores independientes, lo que revela las formas complejas que asumen las estructuras laborales en las empresas contemporáneas. Palabras clave: mercados internos de trabajo, grupos económicos, América Latina. Clasificación JEL: J41, M55, O54.

\footnotetext{
1 Departamento de Estudios Regionales, CUCEA-Universidad de Guadalajara, México. Email: ruben.chavarin@cucea.udg.mx .
}

(C) Revista de Economía Laboral 


\title{
Internal labour markets within business groups in México. A case study
}

\begin{abstract}
This study documents the use of an internal labor market within the commercial chain store $O_{x x o}$, as a part of a business group called FEMSA, which is one of the largest and oldest business groups in Mexico, having operations in other ten Latin American countries. This internal labor market satisfies the next conditions: a) encourages business group own employees' promotion and mobility, including personnel transfers along the business network, becoming a quasi-internallabor market; b) contains organizations created by the business group to develop human capital, includingold-time experiences; and c) shows certain evidence on differences in employees' wages, compared with those of other similar labor options. On the other hand, this case study also shows evidence on the articulation of several labor market structures within FEMSA, where the quasi-internal labor market is combined with personnel subcontracting and participation of independent workers. This pattern reveals the complexity of labor structures used by contemporary firms.
\end{abstract}

Key words: internal labor markets, business groups, Latin America.

JEL Classification: J41, M55, O54. 


\section{Introducción}

Los grupos económicos han dominado extensos sectores de la economía de México a lo largo de décadas, y algunos de estos grupos tienen su origen desde el siglo XIX. Cuando se estudian las razones de la preponderancia de estas redes de empresas suelen señalarse sus capacidades en los siguientes ámbitos: i) resolver en su beneficio fallas de los mercados, ii) establecer condiciones que limitan la competencia económica, iii) lograr un trato privilegiado por parte de distintas agencias gubernamentales, iv) establecer alianzas con empresas multinacionales, y v) obtener sinergias mediante la interacción de diferentes empresas del grupo. Sin embargo, poco se han estudiado los procesos internos que permiten la permanencia y crecimiento de estos grupos.

Uno de los procesos internos más importantes de las empresas se refiere a la forma como éstas asignan el factor trabajo. Bajo condiciones de mercado en donde prevalece la competencia, una empresa común acostumbra contratar a sus trabajadores a través del mercado de trabajo y les asigna un salario que no difiere sustancialmente del que obtendrían en otra empresa desempeñando una labor equivalente. En este tipo de situación se dice que la empresa hace uso de un "mercado externo de trabajo". En cambio, a partir de hace algunas décadas, ${ }^{2}$ se comenzó a documentar el uso de "mercados internos de trabajo" en ciertas empresas de escala grande. Un mercado interno de trabajo puede definirse como aquel mercado donde los trabajadores son contratados externamente para los escalafones básicos (los "puertos de entrada") de la empresa, pero el resto de los escalafones superiores son cubiertos por personal interno de la empresa (Lazear y Oyer, 2004: 528). Como se verá en la sección 2, el uso de este tipo de mercados internos ofrece diversas ventajas a las empresas que los utilizan. Debido a estas ventajas su uso ha sido relativamente común, incluso por empresas anglosajonas que, en teoría, al operar en economías de libre mercado, estarían más apegadas al empleo de los mercados externos de trabajo. Por ejemplo, Groshen y Levine (1998) estudian el caso de 228 grandes empresas localizadas en el medio oeste de los Estados Unidos durante el periodo 1955-1996 y encuentran evidencia de un uso sistemático de mercados internos de trabajo.

Sin embargo, el caso paradigmático del uso de los mercados internos de trabajo ha sido documentado en las redes de empresas

\footnotetext{
2 El estudio de los mercados internos de trabajo data del inicio de los años 1970's, con el trabajo de Doeringer y Piore (1971).
} 
japonesas, conocidas como keiretsu. En este tipo de redes de empresas, las vacantes de puestos de trabajo son cubiertas de manera sistemática por personal interno de la misma empresa, o bien por personal de otras empresas que pertenecen a la misma red de negocios. Como se explica en la sección 2, las transferencias internas de personal en las redes de negocios japonesas son parte de una estructura sociocultural que permite cumplir con una norma social de empleo de por vida (Aoki, 1990; Okabe, 2008). Es por eso que ante diversas circunstancias adversas, o bien ante el propósito de crecimiento de la red, los trabajadores pueden pasar de manera temporal o permanente de una empresa a otra (Imai, 2011).

Los grupos económicos mexicanos tienen cierta semejanza con las redes de negocios japonesas, pero también presentan marcadas diferencias, y definitivamente no persiguen el objetivo del empleo vitalicio para los trabajadores. Sin embargo, aunque el contexto sociocultural sea distinto, podrían usar mercados internos para aprovechar algunas de sus ventajas. El objetivo del presente trabajo es analizar un caso representativo de un grupo económico mexicano y determinar si existe la práctica de formar mercados internos de trabajo en este tipo de redes. En segundo lugar, si es que se está dando este tipo de práctica, ¿cuáles son las características y ventajas de su funcionamiento? En tercer lugar, ¿coexiste el uso de los mercados internos con otros tipos de prácticas para satisfacer las necesidades de demanda de trabajo por parte de estos grupos económicos? El aporte de este trabajo es que ofrece evidencia de un tema que no se ha estudiado en México, ya que en la revisión de literatura no se halló ningún antecedente de este tipo de estudio. La presente investigación también puede servir de referencia para el estudio de este tópico en diversos países de América Latina, ya que en muchos de ellos prevalece un sistema de grupos económicos similar al de México, y tampoco se halló literatura que tratara este tema en otros países de esta región.

Se ha elegido estudiar el caso de la Cadena Comercial Oxxo, que pertenece al grupo FEMSA, uno de los grupos económicos más representativos y antiguos de México (en el año 2015 cumplió 125 años). Este grupo actualmente tiene negocios en la producción de bebidas, tiendas de conveniencia, estaciones de venta de combustible y farmacias, razón por la cual tiene mucha visibilidad pública. Además, tiene operaciones en otros diez países de América Latina y en Filipinas. La elección de un estudio de caso cualitativo se basa en que no existe información pública respecto a los trabajadores (puestos de trabajo, salarios, etc.) de las empresas establecidas en México, lo cual impide hacer estudios de datos en panel o al menos de corte transversal. 
La estructura del artículo es la siguiente. En la sección 2 se presentan algunos enfoques importantes para conceptualizar e interpretar el funcionamiento de los mercados internos de trabajo; en especial se destaca la literatura sobre los keiretsu japoneses, ya que da un tratamiento específico a los mercados internos de las redes de empresas. La sección 3 explica las características principales de los grupos económicos mexicanos y las formas en que podría funcionar un mercado interno en este tipo de red corporativa. La sección 4 corresponde al estudio de caso y consta de siete partes: el primer sub-apartado explica la metodología a seguir en el estudio de caso; el segundo explica cómo se seleccionó el caso; el tercero explica las características del grupo económico FEMSA; el cuarto se enfoca en la cadena de tiendas Oxxo; el quinto muestra la evidencia encontrada sobre los mercados internos de trabajo dentro de la cadena Oxxo y el grupo FEMSA, explicando aspectos como los procesos de contratación y promoción del personal, la presencia de centros de formación de capital humano y los salarios de los empleados directos; el sexto sub-apartado presenta una comparación con otro caso de estudio, con el fin de mostrar alguna peculiaridad del caso OxxoFEMSA; el séptimo se refiere a la posible interacción de los mercados internos de trabajo con otras estructuras del mercado de trabajo dentro del grupo económico. Finalmente, la sección 5 presenta las conclusiones.

\section{Enfoques teóricos sobre los mercados internos de trabajo}

Se puede definir un mercado interno de trabajo con base en tres categorías, aunque diversa literatura sólo emplea dos elementos (Lazear y Oyer, 2004): a) el tipo de contratación de personal en los escalafones altos de la empresa, y b) el tipo de movimiento de los salarios al interior de la empresa. A partir de estos dos elementos, un mercado interno de trabajo se forma cuando los puestos de trabajo de las jerarquías subsecuentes a las más bajas son cubiertos por personal interno y, además, no hay una conexión directa con los salarios del mercado externo.

Una tercera categoría para identificar un mercado interno de trabajo tiene que ver con la presencia de programas de formación de capital humano establecidos por la propia empresa para la formación y desarrollo de las capacidades de los trabajadores y el personal administrativo. En aquellos casos en que se privilegia el uso de un mercado interno de trabajo es común encontrar centros de capacitación y 
entrenamiento formales, e incluso sistemas que llegan a impartir certificaciones, estudios universitarios y programas de posgrado. Estos centros especializados son patrocinados $\mathrm{u}$ organizados por la propia empresa. En diversas redes de empresas de gran tamaño han llegado a crearse centros universitarios con el objetivo de proveer a los negocios de la red de personal educado bajo ciertos estándares y características (Khanna y Yafeh, 2007; Colpan y Hikino, 2010).

Según Toharia (1981), en la creación de los mercados internos de trabajo, además de factores económicos, también intervinieron factores políticos y sociales. Al analizar el caso del surgimiento de estos mercados internos en los Estados Unidos, este autor encuentra que una razón importante de su desarrollo fue impedir la formación de sindicatos en las grandes empresas, o bien reducir su impacto una vez que se formaron. Los mercados internos de trabajo promovieron la estabilidad laboral y la lealtad hacia la empresa, lo que sirvió para diluir el activismo sindical. En contraste, las teorías de corte económico se han centrado en el argumento de que los mercados internos de trabajo son más eficientes que los mercados externos. El propio Toharia (1981: 378) reconoce que estas teorías económicas tienen consistencia lógica y sí pueden explicar los casos empíricos contemporáneos, aunque no proporcionen una explicación adecuada de su desarrollo histórico. Reconociendo esta limitación, en el resto del presente estudio se analizan los distintos enfoques económicos de los mercados internos de trabajo.

La principal razón aceptada en mucha literatura económica se refiere a la preferencia de las empresas por contar con capital humano específico para las características y necesidades de su proceso productivo, según lo señaló inicialmente Becker (1962). La única manera de que los trabajadores adquieran ese tipo de conocimiento específico es trabajar durante un tiempo en la empresa y, de esa manera, ir aprendiendo. Por eso, los trabajadores son contratados desde el mercado externo para los escalafones inferiores (los puertos de entrada) y van ascendiendo a puestos de mayor jerarquía en la medida en que van adquiriendo conocimiento y habilidades. ${ }^{3}$

\footnotetext{
3 Según Toharia (1981: 357), cuando las empresas otorgan formación específica a sus trabajadores les otorgan a estos un poder negociador monopólico, ya que adquieren cualificaciones no disponibles en el mercado externo. Sin embargo, al formar un mercado interno, se limita el poder negociador de los trabajadores "ya que su posible sustituto no es un trabajador externo a la empresa, a quien habría que dotar de todos sus conocimientos específicos (que pueden ser sustanciales), sino el trabajador situado en el escalón inmediatamente inferior que posee una cantidad de conocimientos específicos sólo algo menor."
} 
Una segunda razón importante para el uso de un mercado interno de trabajo es la formación de incentivos para obtener un mejor desempeño de los trabajadores. Según Kräkel y Schöttner (2012), los trabajadores, al poder desenvolverse en un mercado interno y estar protegidos contra la competencia de un mercado externo, obtienen rentas económicas. Las empresas pueden establecer incentivos para lograr un mejor desempeño de los trabajadores con base en las rentas que estos esperan obtener al ascender en la jerarquía de la empresa.

Una tercera razón para el uso de este tipo de mercados es que permiten ahorrar costes debidos a la rotación del personal (Doeringer y Piore, 1971). Este tipo de mercados, al plantear la perspectiva de hacer una carrera dentro de la empresa, permiten retener trabajadores con cualificaciones costosas de reemplazar. Además, al reducirse las tasas de rotación, las empresas obtienen ahorros en sus procesos de reclutamiento de personal.

Una cuarta razón para el uso de un mercado interno de trabajo es solucionar fallas en el mercado de generación de cierto tipo de empleados. Con la creación de los mercados internos las empresas tienen acceso a recursos como insumos o talentos empresariales que escasean en el resto de la economía, y cuya obtención representa altos costos de transacción. Este argumento ha sido utilizado especialmente en la literatura sobre los grupos económicos (Leff, 1978; Li, Ramaswamy y Pécherot-Petitt, 2006; Carney et al., 2011).

Una quinta razón para que las empresas usen este tipo de mercados es que les proporciona una mayor flexibilidad en la explotación de nuevas oportunidades de negocios, y también les permite enfrentar de mejor manera las crisis económicas. En el primero de los casos se necesita personal experimentado para formar nuevas empresas. En el segundo caso puede que una empresa requiera reducir su plantilla laboral y necesite reasignar personal hacia otra empresa, cuando existe una red de negocios (Kim et al., 2004; Cestone et al., 2016).

Hay varios enfoques teóricos sobre el funcionamiento de los mercados internos de trabajo. Sin embargo, la mayor parte de estos tienen como referencia los mercados internos que se forman dentro de una empresa, no dentro de una red de negocios. Además, se centran primordialmente en la formación de estímulos y la determinación de salarios. Waldman (2012) destaca cuatro de estos enfoques, con base en la capacidad de éstos para explicar satisfactoriamente cierta evidencia empírica: i) Aprendizaje simétrico y aseguramiento.- Se basa en el trabajo de Harris y Holmstrom (1982), en cuyo modelo ciertos grupos de individuos que ingresan al mercado de trabajo no pueden ser 
diferenciados entre sí de acuerdo a su habilidad o productividad debido a que comparten algún atributo observable, por ejemplo, su grado de escolaridad. Por otra parte, las empresas intentan "asegurar" a los trabajadores mediante contratos para tratar de evitar que emigren fácilmente hacia otra empresa. ii) Torneos.- Se basa en el trabajo de Lazear y Rosen (1981), cuyo modelo señala que cuando una empresa no es capaz de observar directamente el esfuerzo de cada empleado, establece una competencia (torneo) que le permite premiar y promover al ganador. iii) Promociones como señales.- Se basa en el trabajo de Waldman (1984), el cual establece que una empresa no conoce la habilidad de los trabajadores cuando los contrata. Posteriormente, sólo serán promovidos a puestos de mayor jerarquía aquellos trabajadores que revelan una habilidad mayor que el resto, pero se les tendrá que remunerar de manera tal que no puedan ser atraídos por otras empresas que pueden inferir la habilidad del trabajador a través de la promoción que recibió. iv) Aprendizaje simétrico con adquisición de capital humano y asignación laboral.- Se basa en el trabajo de Gibbons y Waldman (1999), aunque incorpora elementos del trabajo de Becker (1962) sobre capital humano, y del trabajo de Rosen (1982) sobre asignación laboral. En este modelo las empresas no pueden distinguir la habilidad de cada trabajador al contratarlos. Con el paso del tiempo los trabajadores con mayor habilidad innata van acumulando más capital humano que el resto y la empresa puede observarlo y promoverles a un puesto de mayor jerarquía.

Cabe señalar que los cuatro modelos anteriores pertenecen al campo de la economía del personal (personnel economics) (Lazear y Oyer, 2012), que es un enfoque amplio donde se estudian aspectos económicos como las compensaciones e incentivos salariales, el desarrollo de habilidades de los trabajadores, y la organización del trabajo. Aunque a partir de esos cuatro modelos se pueden estudiar los incentivos ligados a las promociones de puestos en cualquier tipo de empresa, no hay ningún tratamiento específico para el análisis de las redes de negocios. Es necesario un enfoque que permita profundizar en el estudio de las redes de empresas, donde hay transferencias de trabajadores entre los diferentes negocios de la red. Este tipo de análisis se encuentra en diversos estudios sobre las redes de empresas japonesas, llamadas keiretsu.

Los keiretsu son redes de empresas formadas por compañías relativamente independientes que tienen tenencias recíprocas de acciones. Estas tenencias cruzadas de acciones se mantienen durante periodos de muchos años, lo que les permite formar relaciones de negocios 
de largo plazo. En este tipo de red los principales accionistas de cada empresa son a su vez otras empresas que suelen ser acreedores, proveedores o clientes de ésta (Hoshi, 1998; Okabe, 2008). ${ }^{4}$ Los keiretsu que presentan más transferencias de personal son los llamados "verticales", caracterizados por tener una empresa manufacturera de gran escala y un conjunto de subsidiarias y subcontratistas que es complementario a esta empresa central de la red. Por ejemplo, las compañías japonesas productoras de automóviles se desarrollaron como keiretsu verticales. Rasgos comunes de este tipo de redes son la cooperación entre proveedores y la compartición de riesgos entre las empresas a lo largo de la cadena vertical de producción (Lincoln y Shimotani, 2010). La complementariedad de los procesos y la cooperación entre proveedores hace que los conocimientos acumulados por los trabajadores puedan ser susceptibles de ser aplicados en diversos negocios de la red, lo que hace factible las transferencias de empleados entre distintas empresas.

Dan (2015) conceptualiza los mercados internos de trabajo de las redes de empresas como "mercados cuasi-internos de trabajo", para diferenciarlos de los mercados internos que prevalecen en una sola empresa de escala grande. En los mercados cuasi-internos los movimientos de personal se dan entre empresas afiliadas a una misma red corporativa. Este mismo autor señala dos razones principales para las transferencias o promociones de personal entre distintas subsidiarias de una red japonesa: a) Para prevenir despidos cuando una subsidiaria necesita ser reestructurada. Debido a que en Japón se estableció una norma social de empleo vitalicio, las empresas de las grandes redes de negocios protegen los empleos de los trabajadores utilizando transferencias de personal temporales o permanentes. Según Jackson (2008) y Keizer (2011), este rasgo de los mercados internos de trabajo se ha mantenido a pesar de las diversas reformas llevadas a cabo en Japón desde la década de los años 1990's con el fin de orientar más la economía hacia el libre mercado. b) Para dotar de personal capacitado y especializado a los nuevos negocios de la red de empresas. Cuando una

\footnotetext{
${ }^{4}$ Según Okabe (2008: 25), en 2006 existían unos 39 millones de accionistas individuales de las compañías japonesas listadas en las bolsas de valores de ese país. Estos accionistas individuales representaban el 90 por ciento de la totalidad de los accionistas. Sin embargo, la cantidad de las acciones en posesión de los accionistas individuales representaba sólo el 18,1 por ciento del total. En contraste, la suma de la tenencia de las acciones de las instituciones financieras (31,1 por ciento), las sociedades no financieras (20,7 por ciento) y las empresas de corretaje (1,8 por ciento) llegaba a representar el 53,6 por ciento del total.
} 
de estas redes quiere expandir sus actividades mediante nuevas subsidiarias, su mercado interno de trabajo les permite incorporar trabajadores de otras subsidiarias que han desarrollado determinados conocimientos y habilidades. Según Hayami, Nakamura y Nakamura (2012), esta conducta incluso aplica cuando las empresas japonesas establecen subsidiarias en otros países y el personal directivo de estas nuevas unidades de negocios es cubierto a través de transferencias procedentes de un mercado interno de trabajo.

Según Dan (2015) hay dos ventajas principales del uso de un mercado cuasi-interno de trabajo: a) las transferencias de personal entre empresas de la red permiten la transmisión de información hacia las empresas receptoras de los trabajadores. Esta información se refiere al know how acumulado en la empresa de la que procede el trabajador, que en muchos casos se trata de un conocimiento complementario al del proceso productivo de la empresa destino. b) Hay un sistema más eficiente para cubrir las vacantes de los puestos de trabajo a lo largo de la red de negocios. El intercambio de información entre las áreas de recursos humanos de las empresas afiliadas simplifica el proceso de reasignación del personal, en comparación con un proceso descentralizado de búsqueda. Este sistema que permite buscar, seleccionar, asignar y entrenar a los trabajadores adecuados para cada puesto de trabajo, ${ }^{5}$ contribuye a mejorar las capacidades de los trabajadores y, por lo tanto, amplifica su contribución a la empresa y a la red de negocios.

El uso de los mercados cuasi-internos de trabajo plantea dos retos principales para las empresas involucradas: i) reunir y procesar la información de recursos humanos que les permita hacer un emparejamiento correcto entre cada trabajador y puesto de trabajo; y ii) establecer mecanismos para lograr que las transferencias de personal satisfagan los requerimientos de reentrenamiento y apoyo para el trabajador, de tal manera que dichas transferencias se puedan efectuar en el tiempo oportuno y de manera eficiente. Los dos retos señalados significan un proceso con cierto grado de complejidad porque, como ya se señaló, en las redes japonesas no hay individuos o familias que tengan la

\footnotetext{
5 Según Aoki (1990: 66), "El sistema de promoción y remuneración de la empresajaponesa ayuda a motivar a sus empleados a desarrollar sus carreras, si no durante toda la vida, sí en una asociación a largo plazo con la empresa. El desarrollo venturoso de la carrera dentro de la empresa es potencialmente muy valioso para el empleado, tanto oficinistas como obreros, porque la paga mensual y los pagos semestrales de bonos aumentan a medida que la posición propia en la jerarquía de rangos mejora con la antigüedad y el desarrollo de habilidades contextuales."
} 
mayoría de la propiedad y el control de las empresas, lo que haría más sencillo el proceso de asignar personal de una empresa a otra.

\section{Grupos económicos en México y mercados internos de trabajo}

Los grupos económicos son la forma dominante de organización de las grandes empresas mexicanas desde finales del siglo XIX. Estos grupos se caracterizan por su sistema de propiedad y control concentrados en familias, donde es común que miembros de estas familias también participen directamente en la operación de los negocios. Otro rasgo que distingue a estas redes de empresas mexicanas son sus negocios diversificados en actividades no relacionadas, así como la integración vertical en las actividades que sí se encuentran relacionadas. Es muy común que las empresas se financien con deuda y no mediante la venta de acciones, lo cual permite que los accionistas controladores mantengan un alto grado de concentración de la propiedad. En los casos en que alguna de las empresas (casi siempre una controladora - holding) participa en el mercado de valores, lo hace emitiendo deuda o acciones con derechos de voto restringidos, lo que contribuye a mantener un sistema corporativo con pocos contrapesos a las decisiones de los accionistas mayoritarios, así como una pobre protección a los accionistas minoritarios (Castañeda, 2010; Chavarín, 2011; y Hoshino, 2010).

Entre los mecanismos de control de los grupos mexicanos contemporáneos sobresale el uso de empresas controladoras. Los accionistas mayoritarios concentran su participación en estas empresas tenedoras que aglutinan las acciones de las empresas subsidiarias, facilitando la coordinación centralizada de toda la red de negocios; en una red de negocios no relacionados es común que existan varias empresas controladoras. Este sistema de coordinación centralizada favorece el intercambio de flujos de recursos entre las empresas de la red de negocios. La gama de recursos que pueden transferirse entre las empresas subsidiarias podría abarcar algunos de los siguientes elementos: a) insumos; b) inmuebles, infraestructura o equipo; c) garantías para obtener créditos; d) créditos relacionados, cuando hay una empresa financiera dentro del grupo; e) préstamos; y f) activos intangibles, como propiedad intelectual. Cabe aclarar que dichas transferencias pueden darse bajo condiciones de mercado, o bien en condiciones más favorables que las de mercado, situación que generaría pérdidas para los accionistas minoritarios (Bertrand et al., 2002); por 
ejemplo, otorgando préstamos o créditos sin solicitar garantías, o cobrando menores tasas de interés que las que se cobrarían a proyectos equivalentes de empresas externas al grupo (Chavarín, 2016).

En teoría, de existir mercados internos de trabajo al interior de los grupos económicos mexicanos, podría facilitarse su funcionamiento a través del sistema de coordinación centralizada dirigido por las empresas controladoras. Mediante estas empresas podría funcionar un sistema de transferencias de personal, ya sea de una subsidiaria a otra, o entre la empresa controladora y una subsidiaria. Cualquiera que sea el caso, se formaría un mercado cuasi-interno de trabajo coordinado por el área de recursos humanos de la empresa controladora.

¿Por qué pueden formarse mercados cuasi-internos de trabajo en un grupo económico? A diferencia de las redes japonesas donde destaca el propósito de reasignar personal para proteger empleos ante las necesidades de reestructuración de los negocios, en los grupos mexicanos destaca el interés por subsanar fallas de mercado que limitan la disponibilidad de trabajo cualificado, ya sea por la falta de cantidad o calidad de compañías de colocación, empresas de búsqueda de talentos, agencias de certificación y escuelas de enseñanza técnica o negocios. Por esta razón, los grandes grupos económicos recurren a fundar sus propios centros de capacitación y educación para formar a sus empleados y promoverlos en sus carreras, rotando y trasfiriendo al personal entre las empresas subsidiarias del grupo, según sea necesario (Colpan y Hikino, 2010; Özgen y Özgen, 2009). Con la creación de los mercados internos, las empresas de los grupos económicos tienen acceso a recursos que escasean en el resto de la economía, y cuya obtención representa altos costos de transacción para empresas que no pertenecen a una de estas redes corporativas (Leff, 1978; Khanna y Palepu, 1999; Murillo y Sung, 2013). Los beneficios de los mercados internos de trabajo aumentan cuando el grupo se encuentra diversificado, debido a la capacidad de estas redes para combinar los recursos necesarios para entrar a nuevos mercados, así como a la flexibilidad que les proveen los mercados internos para hacer frente a las fluctuaciones de la demanda (Kim et al., 2004; Cestone et al., 2016; Faccio y O'Brien, 2017). En las áreas de producción, una ventaja importante sería reducir la rotación del personal $\mathrm{y}$, de manera simultánea, generar incentivos para lograr determinadas metas de productividad del trabajo. En algunos casos, un mercado interno de trabajo también puede otorgarle ventajas competitivas a las empresas de los grupos corporativos en entornos de rigidez laboral, ya que les provee de mayor flexibilidad en la asignación de recursos (Belenzon y Tsolmon, 2016). 


\section{El caso de Oxxo-FEMSA}

\subsection{Metodología de análisis del caso}

En la exposición general del estudio de caso cualitativo se sigue la metodología planteada por Yin (2009), Stake (1999), y VillarrealLarrinaga y Landeta-Rodríguez (2010). Con base en esta metodología se plantean las siguientes preguntas de investigación, formuladas en la seeción 1: i) ¿Existe la práctica de formar mercados internos de trabajo en los grupos económicos mexicanos? ii) Si es que se está dando este tipo de práctica, ¿cuáles son las características y ventajas de su funcionamiento? iii) ¿Coexiste el uso de los mercados internos con otros tipos de prácticas para satisfacer las necesidades de demanda de trabajo por parte de estos grupos económicos? De acuerdo a lo planteado por Yin (2009), se pretende observar si nuestro caso se ajusta a la representación o generalización de un marco teórico. En particular, nuestro estudio de caso permitirá contrastar empíricamente los elementos y funcionamiento de los mercados cuasi-internos de trabajo dentro de un grupo económico, mostrando evidencia sobre tres aspectos: a) el tipo de contratación de personal en los niveles altos de la empresa, b) la formación de organizaciones paralelas establecidas especialmente para desarrollar el capital humano a lo largo de la red de empresas, y c) evidencia de algún tipo de comportamiento particular en la fijación de salarios con relación a los salarios del mercado externo. Adicionalmente, se pretende identificar y, en su caso, mostrar posibles combinaciones de distintas prácticas para satisfacer las necesidades de demanda de trabajo por parte del grupo económico estudiado.

Como se ha señalado desde la sección 1 , se analiza un caso de "tipo único" que se considera importante por tratarse de uno de los grupos económicos más grandes y antiguos de México. Otros argumentos que justifican la selección de este caso se señalan en el apartado 4.2. Para el análisis de nuestro caso se ha recolectado y procesado evidencia documental de diversas fuentes (cuestionarios, uso de reportes de la propia empresa, análisis de notas de prensa, e investigaciones de otros autores) con el fin de aplicar el principio metodológico de la triangulación. Al seguir la metodología de los estudios de caso cualitativos, también exponemos la estructura y funcionamiento de la empresa, así como aspectos relevantes de la historia, desarrollo y estrategias corporativas del grupo económico seleccionado. 


\subsection{Selección del caso de estudio}

Como ya se señaló en la introducción, en México no existe información pública respecto a los trabajadores (puestos de trabajo, salarios, etc.) de las empresas, lo que condujo a la elección de un estudio de caso de tipo cualitativo. Como tampoco hay información sistemática disponible de manera pública para estudiar a cualquier empresa de escala grande en México, se siguió el procedimiento de selección que se describe a continuación. En primer lugar, a partir de la revisión de los trabajos empíricos aplicados en otros países, se elaboró un cuestionario con preguntas para identificar la presencia de un mercado interno de trabajo, dirigido a los gerentes del área de Recursos Humanos de una lista de los 36 grupos económicos de mayor tamaño en México. En segundo lugar, se tomaron en cuenta aquellos casos en donde fue posible obtener el mayor número de respuestas a dicho cuestionario. En tercer lugar, se procedió a considerar los siguientes factores sobre cada grupo: a) la trayectoria histórica, b) los niveles de empleo, y c) la cantidad de información pública disponible. El grupo económico que resultó de este proceso fue FEMSA, el cual, debido a que cotiza en la Bolsa Mexicana de Valores, presenta una buena cantidad de información pública sobre diversos aspectos de su desempeño. Como parte de este grupo económico, resultó relevante la información encontrada respecto a la cadena de tiendas de conveniencia llamada Oxxo.

\subsection{El grupo FEMSA6}

El grupo FEMSA (Fomento Económico Mexicano, Sociedad Anónima) tuvo sus inicios desde a finales del siglo XIX, siendo su primera gran empresa la Cervecería Cuauhtémoc, fundada en 1890. Este grupo económico se diversificó de manera extensa y en forma no relacionada (cerveza, vidrio, petroquímica, siderurgia y servicios financieros) durante gran parte del siglo XX, siendo conocido como Grupo Monterrey y llegando a ser el grupo económico más grande de México. En la década de los años 1970's el grupo se dividió en cuatro partes y la que ahora es FEMSA se volvió un grupo económico independiente llamado VISA, que era el nombre original de la empresa controladora del conjunto de

\footnotetext{
${ }^{6}$ A menos que se especifique otra fuente, la información del estudio de caso procede de los informes y reportes de la empresa controladora FEMSA referidos al final de las referencias bibliográficas.
} 
negocios relacionados principalmente con la producción de cerveza. En 1998 la controladora FEMSA sustituyó a VISA y a partir de entonces esta red fue conocida como grupo FEMSA. En 2010 FEMSA intercambió sus operaciones de la Cervecería Cuauhtémoc Moctezuma por una participación accionaria del 20 por ciento en Heineken a nivel mundial. Una vez que cedió el control de sus operaciones cerveceras, las principales actividades de FEMSA son la producción de bebidas (especialmente los productos de Coca-Cola) en diez países de América Latina y Filipinas, y el comercio al menudeo mediante una cadena nacional de más de 15 mil tiendas de conveniencia (Oxxo), incluyendo una red emergente de tiendas en Colombia. También tiene negocios de estaciones de venta de combustible y farmacias.

Actualmente, la arquitectura corporativa del grupo FEMSA se compone de tres unidades de negocios: 1) Coca-Cola FEMSA, 2) FEMSA Comercio, y 3) FEMSA Negocios Estratégicos. En la primera unidad de negocios se encuentran las subsidiarias de Coca-Cola, Estrella Azul, Santa Clara, Jugos del Valle y AdeS. La segunda unidad de negocios se compone de tres divisiones: a) división comercial, que incluye a Oxxo, Immex, Doña Tota, y Mi Súper Bara; b) división combustibles, que incluye a Oxxo Gas; y c) división salud, que incluye a Farmacias YZA, Farmacias Moderna, Farmacias FarmaCon, Farmacias Cruz Verde, y Maicao. La tercera unidad de negocios engloba a las subsidiarias FEMSA Logística, Imbera, y PTM.

En cuanto a la actual estructura de propiedad y control de los negocios, FEMSA es poseedora del 47,9 por ciento de la propiedad de Coca-Cola FEMSA, mientras que es poseedora del 100 por ciento de la propiedad en cada una las divisiones de FEMSA Comercio (comercial, combustibles y salud); en FEMSA Negocios Estratégicos también posee el 100 por ciento de la propiedad. Por último, como ya se mencionó, FEMSA también tiene una participación accionaria del 20 por ciento en las operaciones internacionales de Heineken. Los accionistas mayoritarios del grupo FEMSA son varios miembros de la familia Garza Lagüera.

\subsection{La cadena de comercio al menudeo Oxxo}

La Cadena Comercial Oxxo pertenece a la división comercial de la unidad de negocios FEMSA Comercio, y fue creada en 1978 cuando se abrió la primera tienda de conveniencia Oxxo en la ciudad de Monterrey, Nuevo León. Esta empresa fue creada con fines estratégicos de comercialización, en específico para la venta de los productos fabricados 
por las unidades de negocio FEMSA Cerveza y Coca-Cola FEMSA. Según datos de diciembre de 2016 la Cadena Comercial Oxxo cuenta con 15.160 tiendas en México, abastecidas a través de 17 centros de distribución. Además, ya hay 65 tiendas localizadas en Colombia.

La organización del trabajo para el funcionamiento de las tiendas Oxxo se divide en dos partes: 1) Una parte operativa, llevada a cabo de dos formas: a) Por "comisionistas mercantiles", que son trabajadores independientes que integran equipos de seis personas, casi siempre compuestos por un jefe de familia y los miembros de la misma, para cada tienda; esta forma prevalece en el 56 por ciento del total de tiendas. b) Por "empleados directos", que son trabajadores propios de la empresa que atienden al restante 44 por ciento de las tiendas. 2) Una parte administrativa, que es desarrollada en 61 oficinas localizadas en 26 entidades a nivel nacional. ${ }^{7}$

\subsection{El mercado interno de trabajo en Oxxo-FEMSA}

De acuerdo a información del 2015, FEMSA Comercio cuenta con 139.205 colaboradores, de los cuales el 56,2 por ciento componen su mercado interno de trabajo, integrado por practicantes, sindicalizados, empleados, gerencia y dirección $(0,3 ; 46,7 ; 8,8 ; 0,4$ y 0,02 por ciento; respectivamente). El restante 43,8 por ciento representa la externalización del trabajo, conjunto formado por los "comisionistas mercantiles" de las tiendas Oxxo, trabajadores por honorarios, y terceros (outsourcing) $\left(43,8 ; 0,0\right.$ y 0,3 por ciento; respectivamente). ${ }^{8}$

\section{humano}

a) Contratación, promoción y centros de formación de capital

El proceso de contratación de los empleados directos inicia con la publicación en la plataforma de la bolsa de trabajo FEMSA de vacantes para puestos de ayudantes de tienda. Quienes ingresan como ayudantes de tienda después pueden ascender a encargados y posteriormente a líderes (véase gráfico 1). Prueba del seguimiento de este proceso de promoción es que el 85 por ciento de los líderes de tienda comenzaron como ayudantes. A su vez, los líderes que cuentan con un alto potencial pueden pasar a cubrir puestos de oficina como entrenador teórico,

\footnotetext{
7 http://www2.oxxo.com/oxxo/oficinas_jalisco.html, consultada el 04 de abril de 2017.

8 Para 2015 no participaban trabajadores por honorarios en FEMSA Comercio y por eso aparece que su participación es de 0,0 por ciento, pero esta cifra puede ser positiva en otros años.
} 
entrenador práctico, asesor comercial, y después poder llegar a jerarquías más altas dentro de la estructura corporativa de Oxxo (véase gráfico 2). ${ }^{9}$ Una característica del proceso de contratación del grupo FEMSA es que el 95 por ciento de los puestos de recién ingreso son cubiertos por practicantes que son elegidos cuando estudian del cuarto al séptimo semestre de su licenciatura. Estos practicantes son los que alimentan el puerto de entrada al mercado interno de trabajo en las áreas de Administración y Finanzas, Expansión y Tecnología, Logística, Comercial, Operaciones, Recursos Humanos, y Planeación y Sistemas. ${ }^{10}$

Sin embargo, en las áreas de producción (para el caso general de FEMSA) y de prácticas comerciales (para el caso particular de Oxxo) el mercado externo de trabajo presenta cierto nivel de desajuste entre las ocupaciones y la cualificación correspondiente de los trabajadores. Por ejemplo, a nivel nacional, los trabajadores industriales que tienen los niveles de instrucción de primaria incompleta, primaria completa y secundaria completa representan el 75,0 por ciento del total, y sólo el 24,9 por ciento restante está compuesto por trabajadores que cuentan con instrucción media superior o superior. Haciendo el mismo análisis para el caso de los comerciantes, aquellos con instrucción igual o inferior a la secundaria completa representan el 54,5 por ciento del total nacional, mientras que aquellos con niveles de instrucción superiores representan el 45,4 por ciento (véase tabla 1). ${ }^{11}$ En estos casos, una función importante del mercado interno de trabajo consiste en mejorar la cualificación de aquellos trabajadores que ingresan a la empresa. Esto también es válido para la formación de cuadros gerenciales especializados, segmento laboral donde a pesar de que los empleados poseen cierto nivel de escolaridad, hay cualidades o habilidades que son escasas y que, por lo tanto, se adquieren dentro del mercado interno de la empresa.

\footnotetext{
9 Véase "Crece con nosotros", en http://www.oxxo.bumeran.com.mx/aplicantes/index.ngmf , consultado el 14 de marzo de 2017.

10 Véase "Red de talentos", en http://www.oxxo.bumeran.com.mx/aplicantes/index.ngmf, consultado el 14 de marzo de 2017.

11 El Sistema Nacional de Clasificación de Ocupaciones (SINCO) provee una clasificación más desagregada de las ocupaciones señaladas. En particular, para el caso de las actividades de FEMSA ligadas a la producción de bebidas, están principalmente los grupos unitarios de "Operadores de máquinas en la elaboración de bebidas alcohólicas y no alcohólicas" y "Trabajadores de apoyo en la industria de alimentos, bebidas y productos de tabaco". En el caso de Oxxo, está principalmente el grupo unitario de "Encargados y supervisores de ventas de productos, servicios financieros y de alquiler". Sin embargo, no existe información de niveles de instrucción a este nivel de desagregación.
} 


\section{Gráfico 1: Mercado interno de trabajo de la Cadena Comercial Oxxo:} empleados directos de tienda.

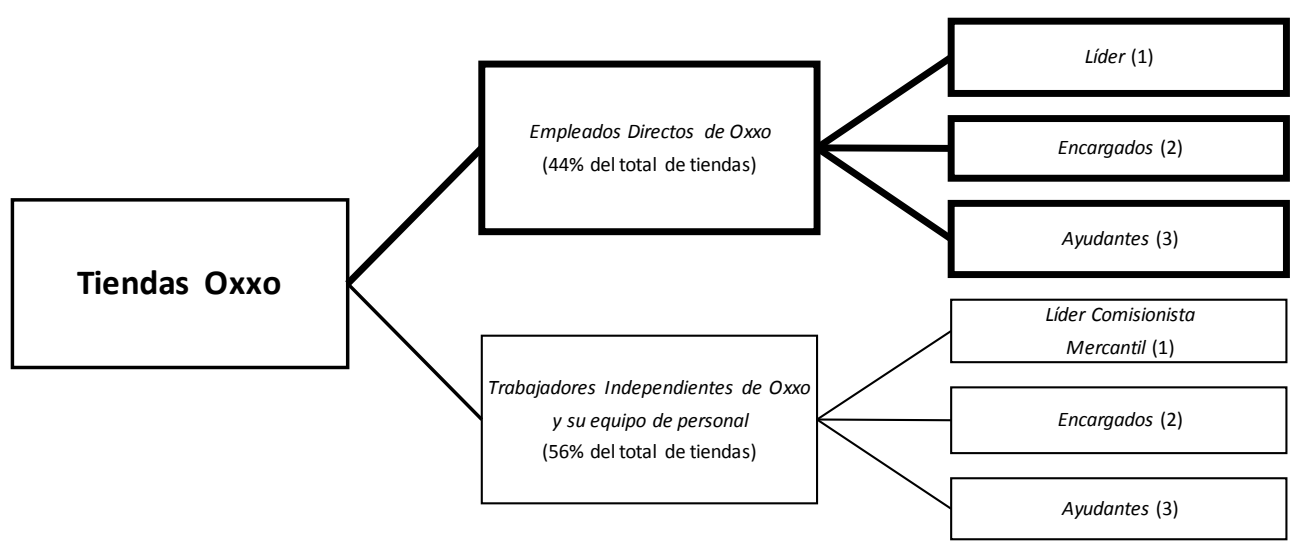

Fuente: Elaboración propia con base en información de: http://www.oxxo.bumeran.com.mx/aplicantes/index.ngmf .

\section{Gráfico 2: Mercado interno de trabajo de la Cadena Comercial Oxxo: área de Recursos Humanos.}

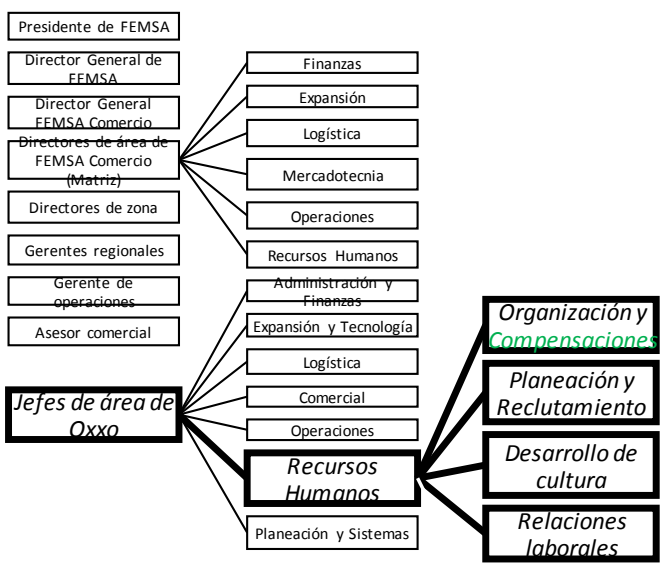

Fuente: Elaboración propia con base en información de: http://www2.oxxo.com/oxxo/oficinas_jalisco.html, y de Zamora (2013). 
Tabla 1: Trabajadores industriales y comerciantes por nivel de instrucción en México, 2017.

\begin{tabular}{lcccc}
\hline \multicolumn{1}{c}{$\begin{array}{c}\text { Nivel de } \\
\text { instrucción }\end{array}$} & $\begin{array}{c}\text { Trabajadores } \\
\text { industriales } \\
\text { (número total) } \\
(*)\end{array}$ & $\begin{array}{c}\text { Porcentaje } \\
\text { de }\end{array}$ & $\begin{array}{c}\text { Comerciantes } \\
\text { (nabajadores } \\
\text { (ndustriales }\end{array}$ & $\begin{array}{c}\text { Porcentaje } \\
\text { de }\end{array}$ \\
\hline $\begin{array}{l}\text { Primaria } \\
\text { incompleta }\end{array}$ & 782.665 & 7,8 & 124.973 & 3,1 \\
comerciantes \\
$\begin{array}{l}\text { Primaria } \\
\text { completa }\end{array}$ & 2.074 .945 & 20,7 & 494.040 & 12,1 \\
$\begin{array}{l}\text { Secundaria } \\
\text { completa }\end{array}$ & 4.652 .231 & 46,5 & 1.608 .654 & 39,4 \\
$\begin{array}{l}\text { Media superior y } \\
\text { superior }\end{array}$ & 2.493 .714 & 24,9 & 1.853 .436 & 45,4 \\
$\begin{array}{l}\text { No especificados } \\
\text { Total }\end{array}$ & 6.490 & 0,1 & 4.475 & 0,1 \\
\hline
\end{tabular}

Fuente: Elaborado con base en información del INEGI, Encuesta Nacional de Ocupación y Empleo (ENOE), IV trimestre de 2017.

Nota: $(*)$ Incluye artesanos y ayudantes.

El grupo FEMSA tiene antecedentes significativos en la creación de una estructura educativa para proporcionar capacitación y desarrollo profesional a sus trabajadores, fortaleciendo su mercado de interno trabajo. ${ }^{12}$ Por la antigüedad de algunos antecedentes se evidencia la intención de subsanar fallas en el mercado de trabajo, ya que en periodos anteriores era notoria la falta de organizaciones especializadas en la formación de capital humano en México. Se destacan las siguientes organizaciones fundadas por este grupo económico: la Escuela Politécnica Cuauhtémoc, creada en 1906; el Instituto Tecnológico de Estudios Superiores de Monterrey (ITESM), en 1943; el Centro Escolar Cuauhtémoc, en 1947; el Instituto FEMSA Comercio y el Instituto FEMSA Logística, ambos en 2000; la Universidad Virtual KOF, en 2004; la Universidad FEMSA, en 2007; el Centro de Biotecnología FEMSA del ITESM, en 2008; el Instituto Oxxo, en 2009. Cabe destacar que el ITESM actualmente es una de las universidades con mayor prestigio académico en México.

\footnotetext{
12 De acuerdo a los Informes de Sostenibilidad del periodo 2013-2016, la inversión en capacitación y desarrollo profesional de los colaboradores del grupo FEMSA ha oscilado entre 1.083 y 1.555 millones de pesos anuales, equivalente a un rango de 59,31 a 85,17 millones de dólares (de USA) de junio de 2016.
} 
De manera específica, para Oxxo se ha establecido la plataforma de formación comercial del Instituto Oxxo, la cual ofrece actualmente dos carreras universitarias en línea para el personal de las tiendas: la Licenciatura en Administración de Empresas Comerciales y la Licenciatura en Gestión de Negocios Comerciales. Además de las carreras, ofrece diplomados, cursos y talleres. De acuerdo a información de 2015, una cantidad de 314 empleados se encontraban becados al 100 por ciento cursando una carrera profesional. De los trabajadores que se han graduado del Instituto Oxxo, el 40 por ciento han sido promovidos a puestos de mayor responsabilidad.

A todo el personal de Oxxo, tanto en los puestos operativos como administrativos, se le entrena en tres diferentes etapas: En la primera etapa, mediante el programa de Inducción o Capacitación, los trabajadores conocen la compañía, las responsabilidades de su puesto de trabajo, los valores, la cultura y operación de la empresa. En la segunda etapa, mediante el Reentrenamiento o Perfeccionamiento continuo, se busca la actualización, profesionalización y productividad del desempeño en las tiendas. En la tercera etapa, centrada en el Desarrollo de los distintos recursos formativos, los trabajadores con alto potencial pueden obtener becas para cursar la preparatoria, carrera profesional, maestría, diplomados o talleres. Las dos primeras etapas son de formación específica y ayudan a reducir los niveles de rotación de personal. La última etapa es de formación general y contribuye a que una parte del personal realice una carrera de largo plazo en la empresa, ocupando puestos de mayor jerarquía en Oxxo o en otros negocios del grupo. ${ }^{13}$ Por ejemplo, los practicantes que son contratados para los empleos de oficina son integrados a ciertos programas de desarrollo como el Programa de becas para maestría, cursos de inglés, la plataforma de aprendizaje de la Universidad FEMSA, o el Programa de talentos en desarrollo. Mediante este último programa el empleado tiene la oportunidad de rotar o ser transferido a otras subsidiarias en otras partes del país, o incluso fuera de éste, a través de asignaciones en otras unidades de negocios como Coca-Cola-FEMSA o FEMSA Negocios Estratégicos.

b) Establecimiento de salarios

13 Informe de responsabilidad social FEMSA Comercio (2015:44) y Tovar (2017). 
En este apartado nos centramos en los ingresos de los empleados directos, ya que estos son los que conforman el mercado interno de trabajo de la empresa. Estos empleados directos son los que operan el 44 por ciento de las tiendas Oxxo, aunque también son empleados directos los que desarrollan las actividades administrativas de la empresa. En particular, se analizan los salarios de los siguientes puestos de trabajo: I) Para los empleados de las tiendas: a) líder, b) encargado, y c) ayudante. II) Para los empleados de oficina en el área de Recursos Humanos: a) generalista, b) reclutamiento y selección, y c) practicante. La tabla 2 muestra los salarios mensuales promedio de estos puestos de trabajo para la cadena Oxxo; la cadena que representa su principal competencia, 7-Eleven; y un promedio de establecimientos dedicados al comercio al menudeo que tipificamos como representativos del mercado externo de trabajo. Los datos representan los salarios mensuales correspondientes al mes de marzo de 2017 y fueron obtenidos de Indeed, que es una empresa subsidiaria de Recruit Holdings Co. Ltd., el líder mundial en sitios web para la búsqueda de empleo.

Tabla 2: Salario mensual promedio (en dólares de USA) de puestos de trabajo seleccionados en el merca do ex terno de trabajo, Oxxo, y 7-Eleven, 2017.

Empleados directos de tienda

\begin{tabular}{lrrr} 
& $\begin{array}{c}\text { Mercado externo } \\
\text { de trabajo }\end{array}$ & $\begin{array}{c}\text { Cadena } \\
\text { Comercial Oxxo }\end{array}$ & 7-Eleven \\
\hline Líder & 441,50 & 584,16 & 499,35 \\
Encargado & 438,03 & 409,67 & 482,73 \\
Ayudante & 207,40 & 209,21 & 213,70 \\
\hline
\end{tabular}

Empleados de oficina del área de Recursos Humanos

\begin{tabular}{lccr}
\hline & $\begin{array}{c}\text { Mercado externo } \\
\text { de trabajo }\end{array}$ & $\begin{array}{c}\text { Cadena } \\
\text { Comercial Oxxo }\end{array}$ & 7-Eleven \\
\hline Generalista & 503,99 & 793,16 & 603,34 \\
Reclutamiento y Selección & 394,02 & 637,79 & 374,47 \\
Practicante & 187,26 & 189,34 & 207,88 \\
\hline
\end{tabular}

Fuente: Elaboración propia con base en información de: www.indeed.com.mx

Nota: Para convertir pesos mexicanos a dólares se tomó el tipo de cambio promedio interbancario a la venta (18,7225 pesos) correspondiente al mes de marzo de 2017.

Según esta información, cuando los empleados están en la parte más baja de la jerarquía laboral, es decir, como ayudantes o practicantes, los salarios no difieren significativamente entre las diferentes opciones 
laborales. En cambio, los incentivos salariales resultan ser más atractivos en niveles más altos de la jerarquía, tanto en las tiendas como en el área de Recursos Humanos, superando los salarios del mercado externo y los de su principal competidor, 7-Eleven. Esto puede tomarse como un indicador de la perspectiva de empleo a largo plazo dentro del mercado interno de trabajo de Oxxo. Cabe señalar que no ha sido posible contar con información desagregada sobre los salarios promedio mostrados, lo que hubiera permitido calcular desviaciones estándar y hacer pruebas estadísticas de diferencia de medias y diferencia de medianas, mismas que hubieran aportado bases más sólidas para establecer diferencias entre los promedios salariales mostrados en la tabla 2.

\subsection{El mercado interno de trabajo en Oxxo-FEMSA comparado con otro caso}

La falta de estudios empíricos sobre los mercados internos de trabajo en México y América Latina impide hacer comparaciones de nuestro caso de estudio con otros casos relevantes, de forma que se identifiquen peculiaridades en el mercado interno de Oxxo-FEMSA. Como un primer ejercicio para poder establecer similitudes y diferencias con nuestro caso de estudio, se hizo una revisión de ciertos aspectos relevantes del Grupo Financiero Santander México (GFSM), que es una de las varias subsidiarias internacionales del Grupo Santander establecido en España.

En primer lugar, GFSM hace un uso mixto de los mercados interno y externo de trabajo para llenar sus vacantes en puestos de media y alta gerencia. Según la propia información de la Bolsa de Trabajo de GFSM, ${ }^{14}$ en los meses de enero a mayo de 2018 un 48,6 por ciento de las vacantes en este tipo de puestos fueron dirigidas hacia candidatos del mercado externo. ${ }^{15}$ El propio director general de Recursos Humanos de Grupo Santander señala que la búsqueda de talento externo a la empresa es una actividad importante y necesaria. ${ }^{16}$ Esta tendencia hacia un

\footnotetext{
14 De acuerdo a datos de 2016 de la Bolsa de Trabajo del Banco Santander México se contrataron desde el mercado externo 1.739 empleados, incluyendo puestos vacantes correspondientes a ejecutivos, gerentes, directores y asesores. Véase GFSM (2016).

15 Véase:

https://santandermx.taleo.net/careersection/ex/jobsearch.ftl?lang=es\&portal=101430233 , consultado el 15 de mayo de 2018.

16 Según José Luis Gómez Alciturri, director general de Recursos Humanos del Grupo Santander: “...,lo esencial es conocer las necesidades del negocio para elaborar los perfiles
} 
mayor uso del mercado externo también se observa en el Grupo Financiero Inbursa (GFI), que es una de las ramas del grupo económico más grande de México (el grupo de la familia Slim), con actividades en telecomunicaciones (en 17 países de América Latina), empresas comerciales, hoteles, empresas de infraestructura y construcción, y servicios financieros, principalmente. En las bolsas de trabajo que promocionan las vacantes del GFI es común observar que se solicitan aspirantes externos a cubrir el puesto de responsable o gerente de sucursal. ${ }^{17}$ Esta diferencia con el caso de Oxxo-FEMSA probablemente se debe a que los mercados externos de trabajo de los servicios financieros se encuentran mejor definidos que en otras industrias, ya que para participar en éstos se requiere de requisitos bastante homogéneos, como haber cursado una licenciatura en el área económico administrativa, poseer alguna certificación del área financiera, y tener cierta experiencia laboral.

En segundo lugar, GFSM también ofrece programas para capacitación y desarrollo de su personal, aunque con dos diferencias respecto a nuestro caso de estudio: i) Su herramienta principal es un programa de posgrado llamado Maestría en Banca y Mercados Financieros, impartido en la Universidad Anáhuac México, en colaboración con la Universidad de Cantabria. Aunque el GFSM tiene el antecedente del Centro de Capacitación Santander, ${ }^{18}$ realmente no hay un paralelismo con la magnitud de los centros de estudio y capacitación creados por el grupo FEMSA. ii) El programa de posgrado señalado no es exclusivo para empleados del GFSM, sino que pueden participar estudiantes de otra procedencia, e incluso empleados de otras empresas financieras.

Como se ha mencionado, las diferencias señaladas son resultado de un primer ejercicio de comparación, aunque se requieren estudios especialmente dirigidos hacia otras empresas para poder comenzar a

que requiere la organización en cada momento. Después, saber dónde están esos profesionales, dentro o fuera de la casa; si es fuera habrá que recurrir a todas las fórmulas posibles para llegar a ellos, incluidas las redes sociales profesionales, como LinkedIn que te permite hacer un zoom sobre todo el talento que existe a nivel internacional y acceder directamente a las personas que necesitas, estén donde estén, incluso sin estar en búsqueda activa de empleo." Véase: Equipos y Talento (2018).

17 Véase por ejemplo: https://www.occ.com.mx/empleos-en-mexico-y-el-mundo/paratrabajar-en-Grupo-Financiero-Inbursa , consultado el 30 de mayo de 2018.

18 El Centro de Capacitación Santander, antes Instituto Santander Serfin, tenía como objeto principal la impartición de cursos y seminarios, conferencias, estudios y eventos de difusión cultural, bursátil y financiera. Hasta diciembre de 2007 dicho Centro se dedicó a las actividades señaladas, pero a partir de esa fecha no ha prestado ningún servicio. 
formular una tipología empírica de los mercados internos de trabajo en las redes de empresas de México y América Latina.

\subsection{Articulación de los mercados internos de trabajo con otras estructuras del mercado de trabajo}

Además del uso de mercados internos de trabajo las empresas pueden usar mercados externos para satisfacer su demanda de trabajo. En principio, el uso de mercados externos significa que los trabajadores de escalafones superiores a los básicos son contratados a partir de la selección de candidatos que proceden de fuera de la empresa o red de empresas, según sea el caso. En este tipo de situación, los trabajadores llegan de un mercado externo pero se integran a la plantilla de la empresa y sus ingresos y prestaciones dependen directamente de esta. Sin embargo, hay otras formas de "externalización del trabajo" que aquí nos interesa destacar y que pueden asumir varias formas (Chavarín, Castillo y Ríos, 1999: 31): i) subcontratación externa (outsourcing), ii) alquiler de personal, y iii) utilización de trabajadores independientes.

El primer caso se da cuando las empresas contratan a otras empresas especializadas en determinadas tareas para que hagan una fracción específica del trabajo con mejores resultados. Entre los servicios más subcontratados se encuentran los de informática, contabilidad, telemarketing, diseño gráfico, investigación, entre otros. En este caso se considera que los servicios o procesos contratados sí se incorporan al producto final de la empresa, (INEGI, 2016:15).

El segundo caso aplica cuando una empresa o institución requiere de servicios que están lejos de la actividad central que desarrolla, por lo que recurre a otra empresa especializada para contratar servicios específicos como jardinería, vigilancia, limpieza, o transporte, entre otros. Estos servicios de apoyo no tienen relación directa con el producto o servicio que se ofrece (INEGI, 2016:16).

El tercer caso incluye dos variantes: a) al personal por honorarios o comisiones, sin sueldo base, que labora por cuenta propia para la unidad económica (excluyendo al personal que no labora de manera regular o exclusiva para la unidad económica); y b) al personal que trabaja para la unidad económica, pero depende contractualmente de otra razón social (excluyendo al personal que trabaja en servicios como vigilancia, limpieza y jardinería) (INEGI, 2016: 16).

En México, en la actividad del comercio al por menor, en específico en las tiendas de autoservicio y departamentales, es común la utilización de trabajadores independientes por pago de honorarios o comisiones. 
Este es el caso de los comisionistas mercantiles de Oxxo. De acuerdo a los datos del censo económico de 2014, se tiene la siguiente información a nivel nacional: En un total de 5.177 supermercados (como Walmart, Chedraui, Soriana) se utilizaron 1.554 trabajadores por honorarios o comisiones, sin sueldo base; mientras que en un total de 36.816 tiendas minisúper (entre ellas Oxxo, 7-Eleven, Circle-K) se utilizaron 53.779 de estos mismos trabajadores. En contraste, en 2.384 tiendas departamentales (como Liverpool, Sears, El Palacio de Hierro) sólo se emplearon 55. Esto implica que las tiendas de minisúper, entre las que se encuentran las tiendas Oxxo, tienden a utilizar en promedio 1,46 trabajadores independientes (por pago de honorarios o comisiones) por tienda (véase tabla 3).

Tabla 3: Personal por honorarios o comisiones de la rama económica "Comercio al por menor en tiendas de autoservicio y departamentales", México, 2013.

\begin{tabular}{lccc}
\hline $\begin{array}{c}\text { Actividad económica por sector } \\
\text { y rama }\end{array}$ & $\begin{array}{c}\text { Número de } \\
\text { unidades } \\
\text { económicas } \\
\text { (UE) }\end{array}$ & $\begin{array}{c}\text { Personal por } \\
\text { honorarios o } \\
\text { comisiones sin } \\
\text { sueldo base } \\
\text { (PHC) }\end{array}$ & $\begin{array}{c}\text { PHC/ } \\
\text { UE }\end{array}$ \\
\hline Comercio al por menor & 1.912 .293 & 115.877 & 0,06 \\
+ En tiendas de autoservicio y & 44.377 & 55.388 & 1,25 \\
departamentales: & 41.993 & 55.333 & 1,32 \\
- En tiendas de autoservicio & 5.177 & 1.554 & 0,30 \\
$\quad$ *En supermercados & 36.816 & 53.779 & 1,46 \\
$\quad$ *En minisúpers & 2.384 & 55 & 0,02 \\
-En tiendas departamentales & & 55 & \\
\hline
\end{tabular}

Fuente: Elaboración propia con base en datos del INEGI-Censo Económico de 2014.

En las tiendas Oxxo el proceso de desarrollo de los comisionistas mercantiles es el siguiente: El área de Recursos Humanos se encarga de la atracción, retención y desarrollo de los colaboradores. Cuando los solicitantes han sido aceptados como comisionistas se les da la capacitación teórico-práctica y durante ese periodo la empresa les paga por el tiempo invertido (Toledo, 2015). Cuando el equipo de un comisionista mercantil comienza a operar una tienda obtienen los siguientes ingresos y prestaciones: comisión por ventas, seguro de vida, seguro voluntario, seguro de gastos médicos mayores, gastos funerarios, caja de ahorro, seguro de auto y becas de estudio. Los comisionistas mercantiles también pueden cursar algunos de los programas formativos 
de FEMSA, siempre que cumplan algunos requerimientos como antigüedad en la empresa y resultados de trabajo en la tienda. ${ }^{19}$

Sin embargo, ampliando el análisis hacia todo el grupo económico, se observa que no sólo se combina el mercado interno de trabajo con el uso de trabajadores independientes, sino que también se emplean otras estructuras del mercado de trabajo. La tabla 4 muestra la distribución de trabajadores por categoría laboral de tres de las áreas principales del grupo FEMSA: Coca-Cola FEMSA, FEMSA Logística y FEMSA Comercio (que incluye a Oxxo). La tabla muestra que al considerar a gran parte del grupo se encuentran aún más diferenciadas las formas de satisfacer la demanda de trabajo. Mientras que FEMSA logística utiliza empleados propios (gran parte de ellos sindicalizados), Coca-Cola FEMSA ha recurrido a una mezcla de empleados propios con outsourcing. ${ }^{20}$ En contraste, FEMSA Comercio, a través de Oxxo, utiliza más trabajadores independientes mediante el modelo de comisionista mercantil.

Tabla 4: Colaboradores del Grupo FEMSA por categoría de trabajo y unidad de negocio, 2015.

\begin{tabular}{lccc}
\hline & Coca-Cola FEMSA & FEMSA Comercio & $\begin{array}{c}\text { FEMSA } \\
\text { Logística }\end{array}$ \\
\hline Dirección & 0,11 & 0,02 & 0,09 \\
Gerencia & 1,28 & 0,35 & 1,01 \\
Empleados & 26,53 & 8,80 & 27,93 \\
Sindicalizados & 58,08 & 46,70 & 69,36 \\
Terceros/outsourci & 13,91 & 0,03 & 0,46 \\
ng & & & \\
Practicantes & 0,00 & 0,30 & 1,16 \\
Comisionistas & 0,00 & 43,80 & 0,00 \\
Total & 100,00 & 100,00 & 100,00 \\
\hline
\end{tabular}

Fuente: Elaboración propia con base en los informes anuales de 2015 de FEMSA. Nota: Los porcentajes mostrados corresponden a los siguientes totales de trabajadores: Coca-Cola FEMSA (100 mil), FEMSA Comercio (139 mil), y FEMSA Logística (14 mil).

\section{Conclusiones}

\footnotetext{
19 http://www.oxxo.bumeran.com.mx/aplicantes/areas-desarrollo.php, consultado el 13 de marzo de 2017.

20 Entre los servicios de outsourcing contratados por Coca-Cola FEMSA se tienen casos como un contrato con la compañía Hewlett Packard para la construcción y operación de su centro de datos (PC World, 2011). Además, están los servicios subcontratados en Colombia para el área de desarrollo comercial en preventa y televenta (El Economista, 2013).
} 
El presente estudio documenta el uso de un mercado interno de trabajo dentro de la cadena de tiendas de conveniencia Oxxo, que a su vez pertenece al grupo económico FEMSA, dedicado a la producción de bebidas, tiendas de conveniencia, estaciones de venta de combustible y farmacias. Este grupo es uno de los más grandes y antiguos de México, y actualmente tiene operaciones en otros diez países de América Latina y Filipinas.

Acorde con la teoría y la evidencia de otras redes de empresas, Oxxo promueve el ascenso y movilidad de sus propios empleados para cubrir las vacantes que surgen en los escalafones superiores de su estructura jerárquica. Esto sucede en la operación de las tiendas y también en las áreas administrativas, pero la movilidad es posible hacia otras empresas del grupo económico, lo que tipifica un mercado cuasiinterno de trabajo. Un segundo aspecto que refuerza este resultado es la presencia de diferencias en los salarios que perciben los trabajadores directos de Oxxo, en comparación con los de otras opciones laborales que operan en el mismo mercado. Un tercer aspecto, que además resulta ser muy significativo, lo constituye la operación de organizaciones establecidas por el propio grupo económico para la formación del capital humano, como lo son la Universidad FEMSA y el Instituto Oxxo. Este tipo de organizaciones se remontan a la Escuela Politécnica Cuauhtémoc, creada en 1906, y el Instituto Tecnológico de Estudios Superiores de Monterrey, fundado en 1943, y que ha llegado a ser una de las universidades más importantes de México. La evidencia analizada en el presente trabajo sugiere que el mercado cuasi-interno de trabajo de esta red de empresas ha buscado sobre todo obtener dos tipos de ventajas: a) resolver fallas de mercado desarrollando capacidades específicas en los trabajadores ante la escasez de esos perfiles en los mercados externos, y b) generar incentivos para el desempeño de los trabajadores ante la perspectiva de ascender en la jerarquía del grupo económico.

Por otra parte, el estudio de caso ha permitido profundizar en el tema de la articulación de distintas estructuras del mercado de trabajo. En específico, en nuestro estudio de caso se encuentran diferenciadas las formas de satisfacer la demanda de trabajo entre distintos segmentos del grupo económico: FEMSA logística utiliza empleados propios; Coca-Cola FEMSA emplea una mezcla de empleados propios con outsourcing; y FEMSA Comercio, a través de Oxxo, utiliza más trabajadores independientes que cobran por comisiones, mediante el modelo de comisionista mercantil. Es decir, el uso del mercado cuasi-interno de trabajo se combina con el uso de subcontratación de personal y el empleo 
de trabajadores independientes, lo que revela las formas complejas que asumen las estructuras laborales en las empresas contemporáneas.

Los resultados de este trabajo ofrecen una primera referencia para el estudio de los mercados internos de trabajo en las redes de empresas de México y el resto de América Latina, ya que en este conjunto de países prevalece un sistema similar de grupos económicos de propiedad familiar.

\section{Bibliografía}

Aoki, M. (1990), La estructura de la economía japonesa, Fondo de Cultura Económica, México.

Becker, G. (1962), "Investment in Human Capital: A Theoretical Analysis", Journal of Political Economy, 70, 9-49.

Belenzon, S. y Tsolmon, U. (2016), "Market Frictions and the Competitive Advantage of Internal Labor Markets", Strategic Management Journal, 37(7), 1280-1303.

Bertrand, M., Mehta, P. y Mullainathan, S. (2002), "Ferreting out Tunnelling: An Application to Indian Business Groups", Quarterly Journal of Economics, 117(1), 121-148.

Carney, M., Gedajlovic, E.R., Heugens, P.P., Van Essen, M. y Van Oosterhout, J.H. (2011), "Business Group Affiliation, Performance, Context, and Strategy: a Meta-analysis", Academy of Management Journal, 54(3), 437-460.

Castañeda, G. (2010), "Evolución de los grupos económicos durante el periodo 1940-2008”, en S. Kuntz (coord.), Historia Económica General de México, El Colegio de México, México, 603-633.

Cestone, G., Fumagalli, C., Kramarz, F. y Pica, G. (2016), "Insurance Between Firms: The Role of Internal Labor Markets", European Corporate Governance Institute (ECGI), Finance Working Paper no. 489.

Chavarín, R., Castillo, V. y Ríos, J.G. (1999), Mercados regionales de trabajo y empresa, Universidad de Guadalajara, UCLA Program on México, y Juan Pablos Editor, Guadalajara. 
Chavarín, R. (2011), "Los grupos económicos en México a partir de una tipología de arquitectura y gobierno corporativos. Una revisión de sus explicaciones teóricas", El Trimestre Económico, 78(309), 193-234.

Chavarín, R. (2016), "Profitability in Banks Affiliated to a Business Groups: Evidence from Mexico", Emerging Markets Finance and Trade, 52(8), 1892-1909.

Colpan, A.M., y Hikino, T. (2010), "Foundations of Business Groups: Toward and Integrated Framework", en A.M. Colpan, T. Hikino y J.R. Lincoln (eds.), The Oxford Handbook of Business Groups, Oxford University Press, 15-66.

Dan, Y. (2015), “The Development of New Businesses and Quasi-Internal Labor Markets in Japanese Firms", Japan Labor Review, The Japan Institute for Labour Policy and Training, 12(2), 28-48.

Doeringer, P.B. y Piore, M.J. (1971), Internal Labor Markets and Manpower Analysis, D.C. Heat and Company.

El Economista (2013), "Coca-Cola Femsa crea empresa de ventas en Colombia", 11 de abril: http://eleconomista.com.mx/industria global/2013/04/11/coca-cola-femsa-crea-empresa-ventas-colombia

Equipos y Talento (2018). "Hay que mejorar la gestión de personas de todos los directivos para que sean líderes de sus equipos", abril: http://www.equiposytalento.com/10-aniversario/entrevistas/directorgeneral-recursos-humanos/banco-santander/jose-luis-gomez-alciturri/21/

Faccio, M. y O'Brien, W. (2017), "Business Groups and Employment”, presentado en la 52 nd Annual Conference of the Western Finance Association, Whistler, Canada.

Gibbons, R. y Waldman, M. (1999), "A Theory of Wage and Promotion Dynamics Inside Firms", Quarterly Journal of Economics, 114, 13211358.

Groshen, E.L. y Levine, D.I. (1998), “The Rise and Decline(?) of U.S. Internal Labor Markets", Federal Reserve Bank of New York. 
Harris, M. y Holmstrom, B. (1982), “A Theory of Wage Dynamics", Review of Economic Studies, 49, 315-333.

Hayami, H., Nakamura, M. y Nakamura, A. (2012), "Wages, Overseas Investment and Ownership: Implications for Internal Labor Markets in Japan", The International Journal of Human Resource Management, 23(14), 2959-2974.

Hoshi, T. (1998), "Japanese Corporate Governance as a System", en K. Hopt, H. Kanda, M. Roe, E. Wymeersch y S. Prigge (eds.), Comparative Corporate Governance: The State of the Art and Emerging Research, Oxford University Press, 847-875.

Hoshino, T. (2010), "Business Groups in Mexico", en A.M. Colpan, T. Hikino y J.R. Lincoln (eds.), The Oxford Handbook of Business Groups, Oxford University Press, 424-455.

Imai, J. (2011), The Transformation of Japanese Employment Relations. Reform without Labor, Palgrave Macmillan, London.

INEGI (2016), Censos Económicos 2014, Personal ocupado subcontratado en las unidades económicas: outsourcing, Instituto Nacional de Estadística y Geografía, México.

INEGI (2017), Encuesta Nacional de Ocupación y Empleo ENOE, IV trimestre.

INEGI (2011), Sistema Nacional de Clasificación de Ocupaciones SINCO.

Jackson, G. (2008), "Employment Adjustment and Distributional Conflict in Japanese Firms", en M. Aoki, G. Jackson y H. Miyajima (eds.), Corporate Governance in Japan. Institutional Change and Organizational Diversity, Oxford University Press, 282-309.

Keizer, A.B. (2011), "Flexibility in Japanese Internal Labour Markets: The Introduction of Performance-Related Pay", Asia Pacific Journal of Management, 28 (3), 573-594.

Khanna, T. y Yafeh, Y. (2007), "Business Groups in Emerging Markets: Paragons or Parasites?", Journal of Economic Literature, 45(2), 331-372. 
Khanna, T. y Palepu, K.G. (1999), "Policy Shocks, Market Intermediaries, and Corporate Strategy: The Evolution of Business Groups in Chile and India”, Journal of Economics and Management Strategy, 8(2), 271-310.

Kim, H., Hoskisson, R.E., Tihanyi, L. y Hong, J. (2004). "The Evolution and Restructuring of Diversified Business Groups in Emerging Markets: The Lessons from Chaebols in Korea", Asia Pacific Journal of Management, 21, 25-48.

Kräkel, M. y Schöttner, A. (2012). "Internal Labor Markets and Worker Rents", Journal of Economic Behavior and Organization”, 84, 491-509.

Lazear, E. y Oyer, P. (2004). "Internal and External Labor Markets: a Personnel Economics Approach”, Labour Economics, 11, 527-554.

Lazear, E. y Oyer, P. (2012), "Personnel Economics", en R. Gibbons y J. Roberts (eds.) Handbook of Organizational Economics, Princeton University Press, 479-519.

Lazear, E. y Rosen, S. (1981), "Rank-Order Tournaments as Optimum Labor Contracts," Journal of Political Economy, 89, 841-864.

Leff, N.H. (1978), "Industrial Organization and Entrepreneurship in the Developing Countries: The Economic Groups", Economic Development and Cultural Change, 26(4), 661-675.

Li, M., Ramaswamy, K. y Pécherot-Petitt, B.S. (2006), "Business Groups and Market Failures: A Focus on Vertical and Horizontal Strategies", Asia Pacific Journal of Management, 23(4), 439-452.

Lincoln, J.R. y Shimotani, M. (2010), "Business Networks in Postwar Japan: Whiter the Keiretsu?", en A.M. Colpan, T. Hikino y J.R. Lincoln (eds.), The Oxford Handbook of Business Groups, Oxford University Press, 127-156.

Murillo, D. y Sung, Y. (2013), "Entender el capitalismo coreano: Los chaebols y su gobernanza corporativa”, ESADEgeo Position Paper no.33.

Okabe, T. (2008), “Capitalismo japonés, ¿modelo económico exitoso o extraño?", Expresión Económica, Universidad de Guadalajara, 21, 19-34. 
Özgen, H. y Özgen, H.M. (2009), "Why Some Types of Business Groups Fall Behind Others? The Case of Regional Networks in Turkey", Selçuk Üniversitesi Sosyal Bilimler Enstitüsü Dergisi, 22, 325-334.

PC World (23 de mayo de 2011), "Coca-Cola FEMSA firma un acuerdo de 100 millones de dólares con HP": http://www.pcworld.com.mx/Articulos/12983.htm, consultado el 14 de diciembre de 2016.

Rosen, S. (1982) "Authority, Control, and the Distribution of Earnings", Bell Journal of Economics, 13, 311-323.

Stake, R.E. (1999), Investigación con estudio de casos, Ediciones Morata, segunda edición, Madrid.

Toharia, L. (1981), "Un test histórico de la teoría de la eficiencia de los mercados internos de trabajo", Cuadernos de Economía, 9(25), 355-380.

Toledo, S.C. (2015), Transformaciones económicas, sociales y culturales en la ciudad de La Paz a partir de la llegada de los Oxxos, Universidad Autónoma de Baja California Sur, Departamento de Economía, La Paz.

Tovar, B.A. (2017), "La teoría del capital humano llevada a la práctica en las ciudades de aprendizaje", Revista de Ciencias Sociales y Humanidades, 51, 45-56.

Villarreal-Larrinaga, O. y Landeta-Rodríguez, J. (2010), "El estudio de casos como metodología de investigación científica en dirección y economía de la empresa. Una aplicación a la internacionalización", Investigaciones Europeas de Dirección y Economía de la Empresa, 16(3), $31-52$.

Waldman, M. (1984), "Job Assignments, Signalling, and Efficiency", Rand Journal of Economics, 15, 255-267.

Waldman, M. (2012), "Theory and evidence in internal labor markets", en R. Gibbons y J. Roberts (eds.) Handbook of Organizational Economics, Princeton University Press, 520-573. 
Yin, R. (2009), Case Study Research. Design and Methods, Sage Publications, Applied Social Research Methods Series, vol.5, $4^{\text {th }}$ Edition.

Zamora, N.S. (2013), Planeación estratégica para disminuir los índices de rotación de personal de tiendas de Oxxo, Instituto Tecnológico de La Paz, División de Estudios de Posgrado e Investigaciones, La Paz.

Páginas electrónicas:

i) $\quad$ www.indeed.com.mx , fecha de consulta: 13 de marzo de 2017.

ii) http://www.oxxo.bumeran.com.mx/aplicantes/index.ngmf , fecha de consulta: 14 de marzo de 2017.

iii) http://www.oxxo.bumeran.com.mx/aplicantes/areasdesarrollo.php , fecha de consulta: 13 de marzo de 2017.

iv) http://www2.oxxo.com/oxxo/oficinas jalisco.html, fecha de consulta: 04 de abril de 2017.

v) http://santandermx.taleo.net/careersection/ex/jobsearch.ftl?lang= es\&portal=101430233 , fecha de consulta: 15 de mayo de 2018.

vi) https://www.occ.com.mx/empleos-en-mexico-y-el-mundo/paratrabajar-en-Grupo-Financiero-Inbursa , fecha de consulta: 30 de mayo de 2018.

Informes anuales, reportes anuales, e informes anuales de sostenibilidad, de sustentabilidad, y de responsabilidad social:

i) Coca-Cola FEMSA (2015), Informe anual. Excelencia en evolución.

ii) FEMSA Comercio, Informe de responsabilidad social 2014.

iii) FEMSA (2015), Informe anual. 125 aniversario 1980-2015, creando historias.

iv) FEMSA (2015), Informe de sostenibilidad. 125 años creando historias de valor.

v) FEMSA (2015), Reporte anual presentado ante la Comisión Nacional Bancaria y de Valores.

vi) FEMSA Comercio (2015), Informe de responsabilidad social. El mejor equipo.

vii) GFSM (2016), Informe de Sustentabilidad. 